\title{
Some elliptic curve based crypto-compression schemes for medical images
}

\author{
Cidjeu D.D. ${ }^{1}$, Tieudjo D. ${ }^{2}$ \\ PhD Student, Department of Mathematics and Computer Science, \\ The University of Ngaoundere, Ngaoundere, Cameroon ${ }^{1}$ \\ Associate Professor, Department of Mathematics and Computer Science, \\ The University of Ngaoundere, Ngaoundere, Cameroon ${ }^{2}$
}

\begin{abstract}
In this paper, we propose two lossless crypto-compression schemes based on Elliptic Curve (EC) for the security of medical images. Pixels values are computed as elements of a finite field $F q$. For compression, these elements are grouped in blocks in an appropriate manner. After transforming these blocks into points of an elliptic curve, two EC-based encryption schemes are applied. We obtain two crypto-compression schemes which compared to some existing systems, offer better performances in terms of compression rate, image quality and execution time.
\end{abstract}

Keywords: Image, Crypto-compression schemes, Elliptic Curve Cryptography, Discrete Logarithm Problem (DLP).

\section{INTRODUCTION}

Elliptic Curve Cryptography (ECC) has performed remarkably well in recent years. Indeed, operations on elliptic curves are faster and more efficient for the implementation of cryptosystems [1]. In addition, for an equivalent level of security, cryptosystems based on elliptic curves require smaller key sizes than those based on Number Theory. For example, a security level of 80 bits is reached with 160-bit keys with ECRSA against 1024 bits for RSA [5]. This makes EC an appropriate platform for developing cryptographic systems. Many cryptosystems based on EC have been developed. Among them we have encryption schemes such as Elliptic Curve Integrated Encryption Scheme (ECIES) [5][11], ElGamal's analog [9] and Massey-Omura's analog [9]; key agreement protocols like Elliptic Curve DiffieHellman (ECDH) key exchange [5]; digital signatures protocols like Elliptic Curve Digital Signature Algorithm (ECDSA) and Edwards curve Digital Signature Algorithm (EdDSA) [5]; pseudo-random generators [5], etc.

A crypto-compression scheme is a hybrid process that reduces the size of an image while ensuring its confidentiality. There are many crypto-compression schemes in the literature. In 2006, Puech and al. proposed a crypto-compression scheme based on AES encryption and DCT compression [15]. But the DCT compression brings remarkable losses on the image from a certain level of compression rate [4]. So, Benabdellah and al. proposed in 2006 and 2007 two cryptocompression schemes based on Discrete Wavelet Transform (DWT). The first scheme uses DES [2] while the second uses AES [3]. These two schemes use encryption based on Number Theory (AES and DES), which is said will be made vulnerable by the quantum computer. Furthermore, Number Theory based cryptosystems generally need big key size to be secured, which is a problem in practice. The execution time needed in decompression is also a limit in schemes based on DWT. Another platform, chaos-based cryptography, has also been used for image crypto-compression ([8], [11]). However, although it is fast, it lacks robustness and good security, as mentioned in [18]. Crypto-compression schemes are evaluated by the image quality after reconstitution, the compression rate, the execution time and the security level. Having a fast cryptosystem which guarantees more security and offers better quality and better compression rate is still a challenge and a real need, precisely for medical images, where good security, higher image quality and small execution time are all required. Moreover, as we know, there is no image crypto-compression scheme based on EC.

In this paper, we propose two lossless crypto-compression schemes based on EC. Given an image $I$, a finite field $F q$ and an elliptic curve $E$ over $F q$. For compression, we group $I$ in $8 \times 8$ pixels blocks. For each row of any block $B$, we compute an element of $F q$ which represents that row. The next step consists of transforming the obtained elements of $F q$ into points of the elliptic curve $E$. Then, two EC-based encryption schemes (Massey-Omura and ElGamal) are applied to encrypt the points of $E$ representing $I$. This provides two crypto-compression schemes. Applied to some medical images, the results obtained are more efficient in terms of image quality, compression rate (weight) and speed (execution time), compared to some existing in the literature 


\section{Preliminaries}

\section{A. EC based encryption}

Encryption with elliptic curves has been presented in [9]. We describe below two encryption schemes based on EC. In these cryptosystems, a message is seen as a point of an elliptic curve.

\section{A.1. Massey-Omura's encryption scheme}

Given an information represented by a point $P_{m}$ of a given elliptic curve, the encryption of $P_{m}$ can be performed with the scheme described below.

\begin{tabular}{|l|l|}
\hline Public parameters & $\begin{array}{l}E, \text { an elliptic curve over a finite field } F q \\
N=\# E\end{array}$ \\
\hline Alice Private key & $\begin{array}{l}e_{A} \in[1, n], \text { such that } \operatorname{gcd}\left(e_{A}, N\right)=1 \\
d_{A}=e_{A}^{-1} \bmod N\end{array}$ \\
\hline Bob Private key & $\begin{array}{l}e_{B} \in[1, n], \text { such that } \operatorname{gcd}\left(e_{B}, N\right)=1 \\
d_{B}=e_{B}^{-1} \bmod N\end{array}$ \\
\hline \multicolumn{2}{|c|}{ Alice want to send to Bob a message which is a point of $E, P_{m}$} \\
\hline Encryption & $\begin{array}{l}\text { Alice send to Bob } e_{A} P_{m} \\
\text { Bob send to Alice } e_{B} e_{A} P_{m} \\
\text { Alice send } P_{m}^{\prime}=d_{A} e_{B} e_{A} P_{m}\end{array}$ \\
\hline Decryption & Bob compute $d_{B} P_{m}^{\prime}=P_{m}$ \\
\hline
\end{tabular}

Figure 1. Encryption/decryption by Massey-Omura's scheme [9]

This scheme is built on the difficulty to solve the Discrete Logarithm Problem [9]. If one can solve this problem, then he can compute $e_{A}$ and $e_{B}$ and then recover the message.

\section{A.2. ElGamal's encryption scheme}

A message seen as point of an elliptic curve can also be encrypted by the ElGamal's analog presented here.

\begin{tabular}{|l|l|}
\hline Public parameters & $\begin{array}{l}E \text {, an elliptic curve over a finite field } F q \\
\text { A base point } B\end{array}$ \\
\hline Bob Public key & $a B$, where $a$ is an integer \\
\hline Bob Private key & The integer $a$ \\
\hline \multicolumn{2}{|c|}{ Alice want to send to Bob a message which is a point of $E, P_{m}$} \\
\hline Encryption & $\begin{array}{l}\text { Alice chooses an integer } k \text { and send to Bob the } \\
\text { pair of points }\left(k B, P_{m}+k a B\right)\end{array}$ \\
\hline Decryption & Bob compute $P_{m}+k a B-a k B=P_{m}$ \\
\hline
\end{tabular}

Figure 2. Encryption/decryption by ElGamal's scheme [9]

Once again, the Discrete Logarithm Problem is the base of the security of the scheme. By solving it, one can compute $a$ and recover the message.

For these cryptosystems to be applied on images, a transformation of image into points of elliptic curve is needed. This transformation is described in the next section.

\section{B. EC transformation of images}

Let $I$ be an image. In this section, a process to transform $I$ into a sequence of points of an elliptic curve is presented. Each pixel will be seen as a point of a given elliptic curve. Any pixel $P_{m}$ can then be encrypted by the encryption schemes presented previously.

\section{B.1. Transforming a character to a point of an elliptic curve}

For ECC to be applied directly on any data, these data have to be transformed into points on elliptic curve. In [9], Koblitz described a process to transform a character into a point of an elliptic curve. A character is seen as an integer $m$, such that $0 \leqslant m \leqslant M \in N$. For example, letters (A to Z) are numbers between 0 and 25 . For a given character $m$, 
Vol. 8, Issue 5, May 2019

Algorithm 1 below computes a pair (x,y) which is a point of an elliptic curve, representing the given character. Assume that we have a finite field $F q$ such that $q$ is on the form $q=p^{r}, p$ prime, $r>0$; and $q \geqslant M k+1$, where $k$ is generally set to 30 or 50. Given the curve $y^{2}=x^{3}+a x+b$ over the finite field $F q$ and given a character represented by an integer $m$.

Compute for each $j=1, \ldots, k$,

$$
m k+j
$$

Let $x$ be the corresponding element of $m k+j$ in $F q$.

For such $x$, we compute $y^{2}=f(x)=x^{3}+a x+b$ and find a square-root for $f(x)$. If there exists a $y$ such that $y^{2}=f(x)$, the point of the elliptic curve representing $m$ is $P_{m}=(x, y)$. If there is no square-root for $f(x)$ for the current $j$, we jump to the next $j$. With $k=30$ or $k=50$ the algorithm always returns a good result [9]. This process is detailed in Algorithm 1 .

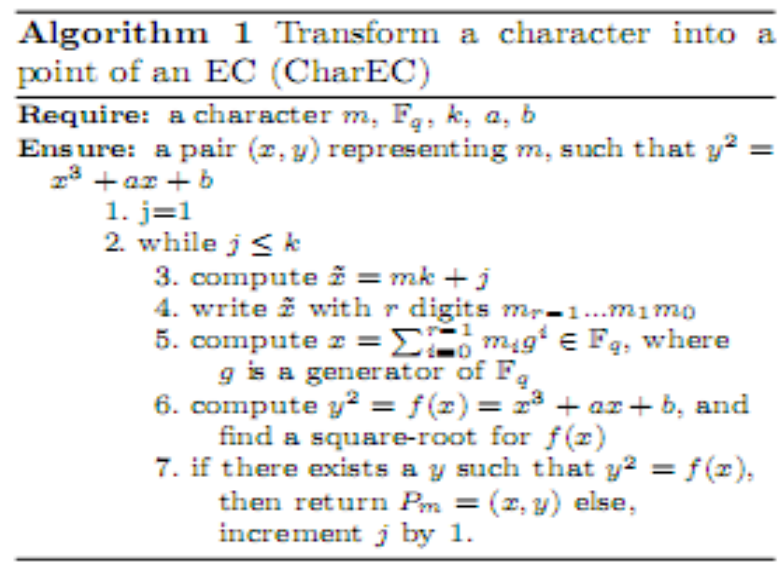

From Algorithm 1, given a point $(x, y)$ representing a character, this initial character $m$ can be recovered by computing $\left\lfloor\frac{(\tilde{x}-1)}{k}\right\rfloor$, where $\lfloor v\rfloor$ represents the integer part of $v$ and $\tilde{x}$ is the integer which corresponds to $x$ in the equivalence between the integers and the elements of $F q$.

\section{B.2. Transforming image to points of an elliptic curve}

Algorithm 1 above describes how to transform a character into a point of an elliptic curve. An image is a sequence of integer values between 0 and 255. We present below how to generate the sequence of points of an elliptic curve representing a given image. Algorithm 2 shows how the integer values between 0 and 255 are represented as points of a given elliptic curve.

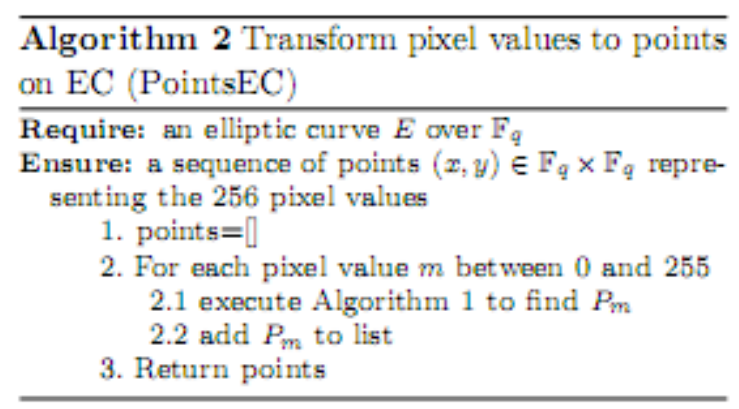

Using algorithm 2, it is shown below (Algorithm 3) how to obtain a sequence of points of an elliptic curve, representing a given image. 


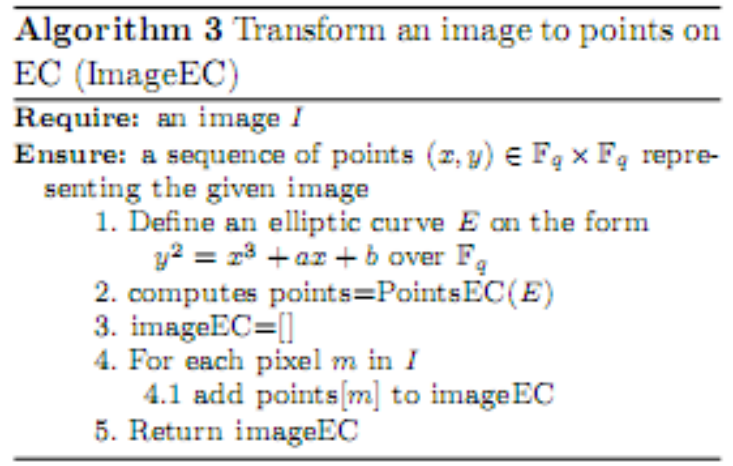

By using Algorithm 3, an image can be viewed as points of an elliptic curve, fixed in advance.

Consequently, what is done on images can be done on points of an elliptic curve and what is done on elliptic curves can be applied on images.

\section{Image crypto-compression}

In medical applications, the size of scanned images is usually very big. Compression is therefore used to improve storage capacity and reduce the transmission time of medical images across networks. Some known compression approaches are the Discrete Cosine Transform (DCT) based compression and the Discrete Wavelet Transform (DWT) based compression. So, to secure images, encryption is generally joined to compression to obtain a hybrid process called crypto-compression, which produces a compressed and encrypted image, as described in Figure 3 below.

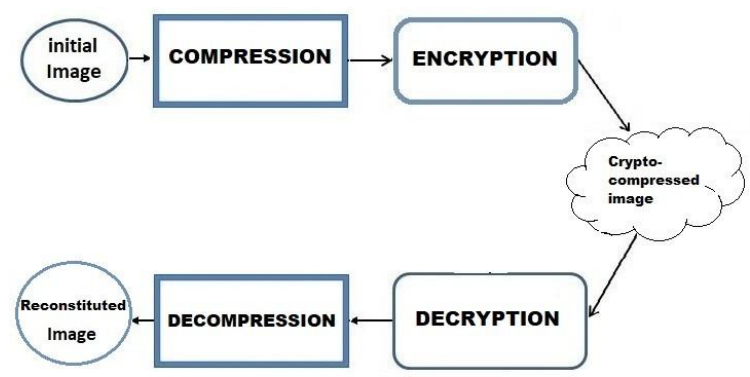

Figure 3. Classical approach to crypto-compression

The scheme takes an image, compresses it and then encrypts certain compression parameters so that the cryptocompressed image is not decipherable. When the encrypted parameters are decrypted and decompression applied, we obtain the reconstituted image [4].

\section{PROPOSED IMAGE CRYPTO-COMPRESSION SCHEMES}

\section{A. Image crypto-compression}

\section{A.1 General description}

Let $I$ be an $L \times l$ image. Assume $I$ is a colour image. Then, $I$ is initially represented by a $L \times(l \times 3)$ byte matrix. The proposed crypto-compression schemes have three main phases:

1. Block compression and decompression presented in section III.A.1.1,

2. EC transformation and inverse transformation (section III.A.1.2),

3. Encryption and Decryption (section III.A.1.3). 


\section{A.1.1. Block compression/decompression}

Let $I$ be a colour image of $L$ lines and $l$ columns. Each pixel is coded by 3 values from 0 to 255 . Let $M$ be the $L \times(l$ $\times 3$ ) bytes matrix representing $I$. For all $i$ and $j$ such that $0 \leq i<L$ and $0 \leq j<l \times 3$, we have $0 \leq M(i, j)=x_{i, j} \leq 255$. The block compression reduces every $8 \times(8 \times 3)$ pixels block of the image to a $8 \times 3$ block of element of $F p$ as described below.

Let divide $M$ into $8 \times 8$ blocks of pixels. Let $B=\left(x_{i, j}\right)_{0 \leq i<8,0 \leq j<8 \times 3}$ be the $8 \times(8 \times 3)$ bytes matrix representing a $8 \times 8$ pixels block of $M$.

For every row $i$ in $B$, compute

$$
e l t_{i}=\left(e l t_{i, 1} ; \text { elt }_{i, 2} ; e l t_{i, 3}\right)
$$

where

$$
\text { elt } t_{i, t}=\sum_{j=s(t-1)}^{s t-1} x_{i, j} \times\left(10^{3}\right)^{8 t-j-1}
$$

We obtain

$$
\begin{aligned}
& \text { elt }_{i, 1}=\sum_{j=0}^{7} x_{i, j} \times\left(10^{3}\right)^{7-j} \\
& \text { elt }_{i, 2}=\sum_{j=8}^{15} x_{i, j} \times\left(10^{3}\right)^{15-j}
\end{aligned}
$$

and

$$
e l t_{i, 3}=\sum_{j=16}^{23} x_{i, j} \times\left(10^{3}\right)^{23-j}
$$

The values of $B$ are computed as elements of a finite field $F p$. Let $F p$ be a finite field such that $p$ is prime and $p \geqslant\left(12^{24}\right)$ $\times k+1$, where $k$ is set to 50 or 30 ; let $E$ be an elliptic curve over $F p$. Since the $x_{i, j}$ are from 0 et 255 and $p \geqslant\left(10^{24}\right) \times k$ +1 , then elt $_{i}=\left(\right.$ elt $_{i, 1} ;$ elt $_{i, 2} ;$ elt $\left._{i, 3}\right) \in F_{p}^{3}$. So, elt $t_{i}$ is a triple of elements of $F p$ representing line $i$ of $B$. By this operation, we move from a matrix $B$ of size $8 \times 3 \times 8$ to a matrix $B^{\prime}$ of size $8 \times 3$. The obtained block $B^{\prime}$ are merged to form a matrix $M^{\prime}$ of size $L \times\left\lceil\frac{l}{8}\right\rceil$, where $\lceil x\rceil$ is the first integer greater or equal to $x$. The block compression is performed by Algorithm 4.

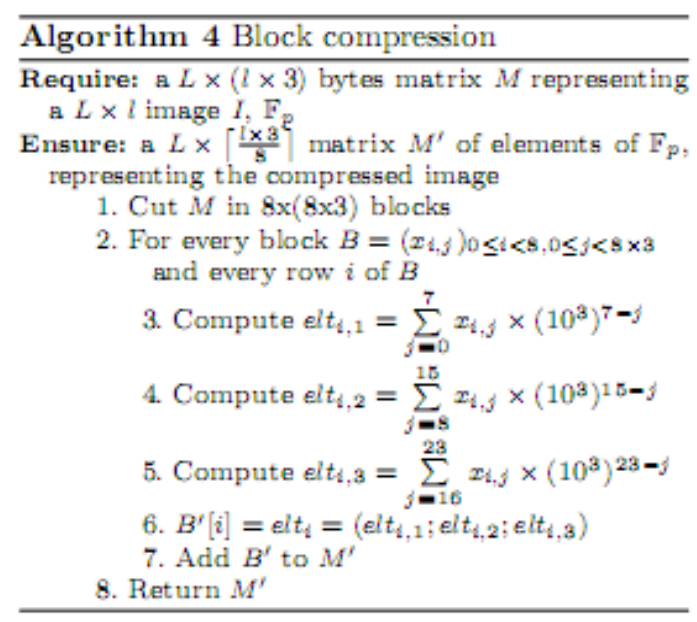

By Algorithm 4, a compression ratio of $1-\frac{1}{8}=87.5 \%$ is performed. 
Vol. 8, Issue 5, May 2019

\section{A.1.2. Elliptic Curve Transformation/Elliptic Curve Inverse Transformation}

At this step, we are transforming a matrix M' of elements of a finite field $F p$, representing a compressed image, into a matrix of points of an elliptic curve. Assume that we have a finite field $F p$ such that $p$ is prime and $p \geqslant(1025) \times k+1$, where $k$ is generally set to 30 or 50 . Given the curve $y^{2}=x^{3}+a x+b$ over the finite field $F p$ and given an image $I$ represented by a matrix $M^{\prime}$ of elements of $F p$. For every value $m$ in $M^{\prime}$, Algorithm 1 is used to compute the point of $E$ representing $m$. Every point of $M^{\prime}$, which is a block of 8 pixels, is then replaced by a point of $E$. This process is described in Algorithm 5 below.

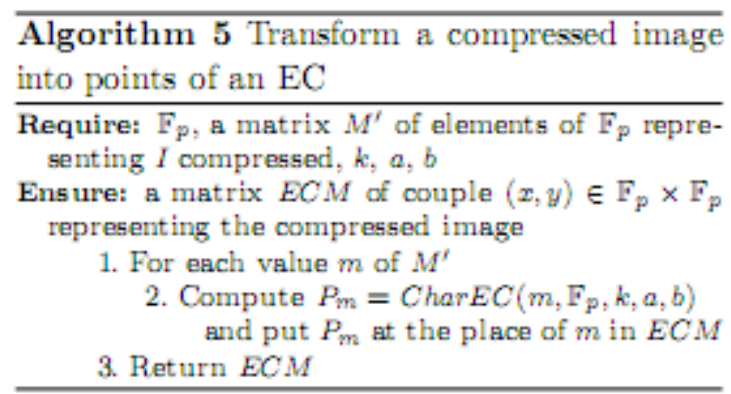

\section{A.1.3. Encryption/Decryption}

The encryption schemes used in this step are those presented in sections II.A.1 and II.A.2. The application of these two encryption schemes on compressed images seen as points of an elliptic curve leads us to the two crypto-compression scheme presented in the next sections.

\section{B. The Massey-Omura type scheme}

Here, the points representing the compressed image are encrypted by the Massey-Omura encryption scheme.

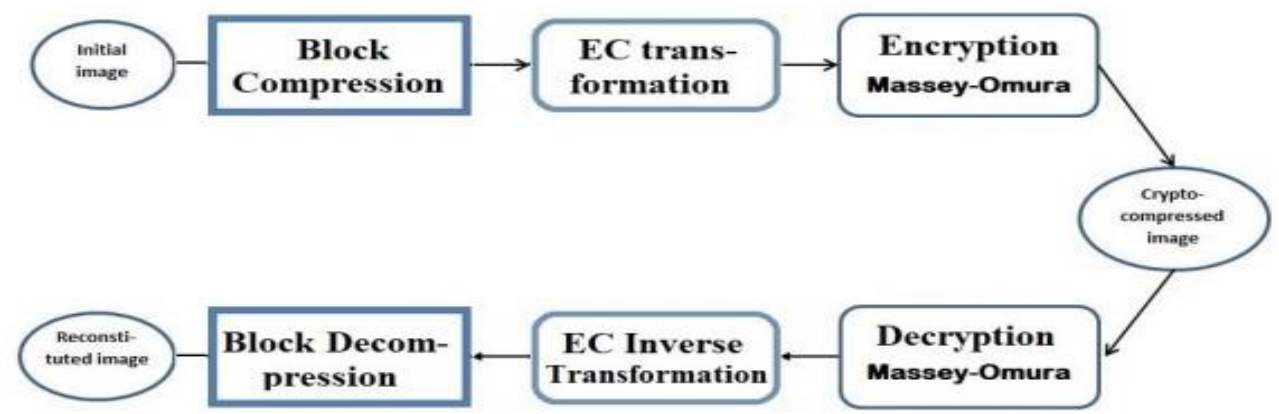

Figure 4. Crypto-compression scheme based on Massey-Omura encryption

\section{The ElGamal type scheme}

Here, the ElGamal encryption scheme is used to encrypt the points representing the compressed image.

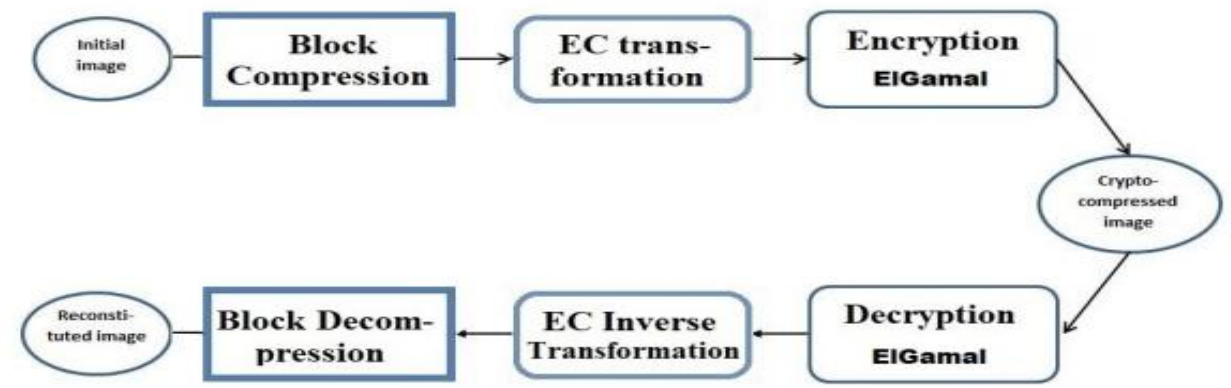

Figure 5. Crypto-compression scheme based on ElGamal encryption 
Vol. 8, Issue 5, May 2019

\section{IMPLEMENTATION, RESULTS AND DISCUSSION}

In this section, the Massey-Omura and ElGamal based schemes are applied on the image "lena". We illustrate the implementation. From the recovered images after implementation, we compute the image quality and the compression rate. The results are compared to some existing crypto-compression schemes: JPEG-AES, FMT-AES and JPEG-LFSR.

\section{A. Illustrations}

Let $I$ be the image presented in figure 6 .

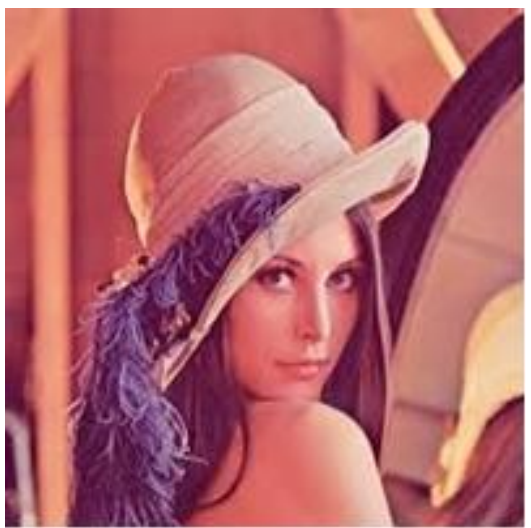

Figure 6. Sample image "lena"

Let $B$ be the $8 \times(8 \times 3)$ pixels block below, extracted from $I$ (Figure 7$)$.

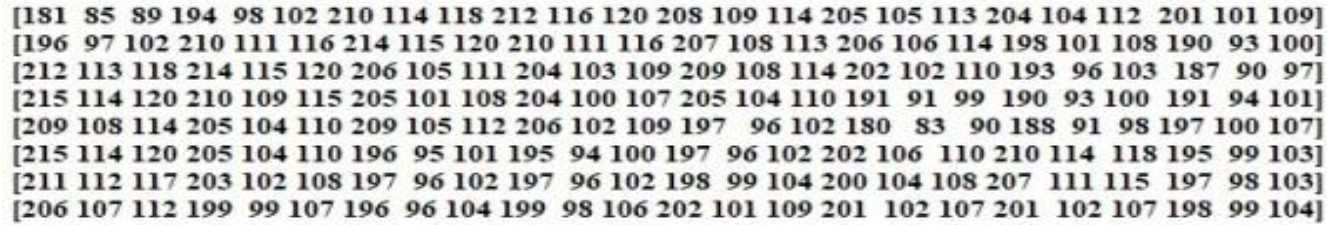

Figure 7. An $8 \times(8 \times 3)$ pixels bloc extracted from I

The elliptic curve defined by

$$
\begin{gathered}
y^{2}=x^{3}+8607703086069211603740389 * x+3944753409163852864976165 \text { over the finite field of size } \\
p=9194940935662755805691087
\end{gathered}
$$

has been generated.

The block compression of $B$ gives $B$, the $8 \times 3$ matrix presented in figure 8 . The elements of this matrix are all in $\mathrm{Fp}$. The first element, compute from the first 8 pixel values of $\mathrm{B}$ is

$$
\text { elt }_{0,1}=181085089194098102210114 \text {. }
$$

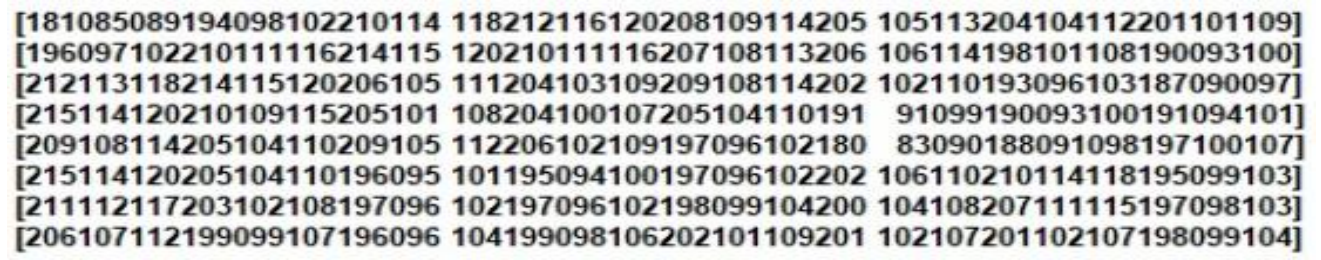

Figure 8 . The $8 \times 3$ matrix obtained after row compression 
Vol. 8, Issue 5, May 2019

The sequence of points of $E$ representing $B$ ' is shown on figure 9.

[I(5432552675822943066303421: 2181074533967633087284250:1), (3546363483606243273426150: 298437797364159101508638: 1), (3153396123123366033033271 : $77641192646256252654697: 1)]$, [(5882913066303333486423450: $3544334886326394563167469: 1)$, (3606303333486213243396180 : 808584977369673217366137: 1), (3183425943033245702793000 : $1634707051192673592790909: 1)]$, [(6363393546423453606183150: $140303416078458529993641: 1)$, (3336123093276273243426064 : $2573772106825497898723330: 1)$, (3063305792883095612702910 : 863533335479157004799454: 1)], [(6453423606303273456153030: $1145690416667479881785989: 1)$, (3246123003216153123305730 : $3337073052674808546802738: 1)$, (2732975702793005732823035: $150047494182361002398824: 1)]$, [(6273243426153123306273152: $3115488627365979385578692: 1)$, (3366183063275912883065400 : 2931432536768990021556017: 1), (2492705642732945913003210 : $1951224737429512321435284: 1)]$, [(6453423606153123305882850: $3671227255711398080608933: 1)$, (3035852823005912883066060 : 20719707193914097368399: 1), (3183306303423545852973090:2363483130193579751119724:1)], [(6333363516093063245912880: $1619542044935651323570932: 1)$, (3065912883065942973126000 : $178293515027268779112948: 1)$, (3123246213333455912943090:4193464944833841470814307:1)], [(6183213365972973215882881: $494038131959205661519572: 1)$, (3125972943186063033276032 : $4537586987392051618798923: 1)$, (3063216033063215942973121 : $3624192400884451079634437: 1)$ ]

Figure 9. Sequence of points representing the bloc

Figure 9 presents points as triples $\left(X_{P}: Y_{P}: Z_{P}\right)$, which are their projective coordinates, according to the affine coordinates $\left(X_{P} / Z_{P}, Y_{P} / Z_{P}\right)$ if $Z_{P}$ is non-zero, and $O$ (the point at infinity) if $Z_{P}$ is zero.

For the first point for example, $P_{m}=(5432552675822943066303421: 2181074533967633087284250: 1)$, if we compute

$$
\frac{5432552675822943066303421-1}{k}
$$

for $\mathrm{k}=30$, we obtain 181085089194098102210114 which is the first element of the compressed block. So, the inverse transformation is assured.

This matrix representing the compressed block is a $8 \times 3$ matrix of points instead of $8 \times 8 \times 3$. This constitutes a compression rate of $1 / 8$. When the above sequence of points is encrypted with the Massey-Omura (MO) encryption scheme described in section II.A.1, we obtain the following sequence of points (Figure 10).

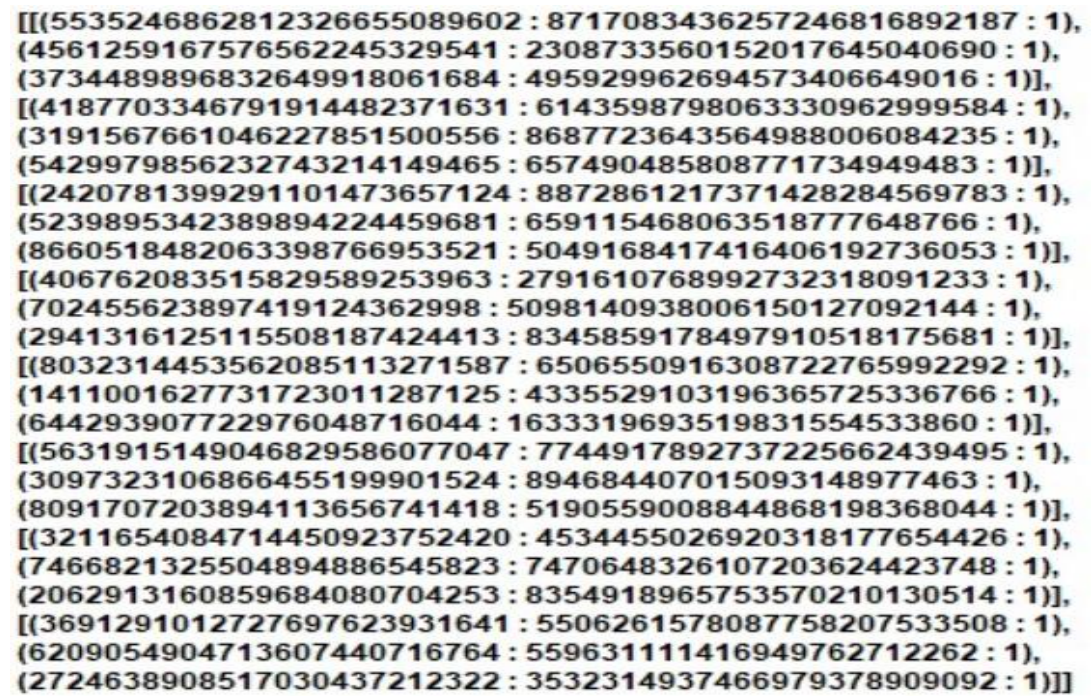

Figure 10. Sequence of points representing the block encrypted with Massey-Omura encryption 
Vol. 8, Issue 5, May 2019

When the block is encrypted with the ElGamal encryption scheme described in II.A.2, the sequence of points shown on Figure 11 is obtained.

\begin{abstract}
[I(214060464270854392426516: $852562785847274658450317: 1)$, (2862012023692007466625438: $232076589880063226590751: 1)$, (9108131462754502592040240: 8312306081162339731390307:1)], [(1985984868415909361384719:5830766085361424523447976:1), (302537185517544044952500: $2578985092809310205224457: 1$ ), (8114472708360581098857451: $4098482905088903880067365: 1)]$, [(2135662420723279877380716:7861932038320269287726922:1), (422999846642281436549628: $6231533479965521579000623: 1)$, (3845433008519229927268286: $1752954519719484136523616: 1)]$, [(730964982242977702549822: $4346598127426425501746538: 1)$, (8395426931148273714837098: $8233658225475306535030750: 1)$, $(6938087571552847815024084: 7685853814046644288993814: 1)]$, [(4390366661324957784710662: 2378217254457109855097816:1), (2087085422695338853387540: $16473031781733695555816: 1)$, (3628495081788474939866025: $871155951985339119028924: 1)]$, [(4194111991746713430187833: $3068852350088253732866910: 1)$, (4833113760022328561755870: $4838587234134247241854201: 1)$, (295132881081133745942114: $5881804348035801022395518: 1)]$, [(4041044955985864962471093:3622340234424706755762192: 1), (2061891433420139067916877: $959262852793019705853790: 1)$, (794612443927762773684277: $5591055188785520929230715: 1)]$, [(6914513020402061599250061: $3610499576760741696941475: 1)$, (4619976938623673782388880 : $1790207512146762294638344: 1)$, (4954023561976283752588974: $5943106358029598722096095: 1)]$
\end{abstract}

Figure 11. Sequence of points representing the block encrypted with ElGamal encryption

The decryption of the two encrypted blocks returns the initial values, as we can see on figure 12 .

[[(5432552675822943066303421: $2181074533967633087284250: 1)$, (3546363483606243273426150: 298437797364159101508638: 1), (3153396123123366033033271: $77641192646256252654697: 1)]$, [(5882913066303333486423450: $3544334886326394563167469: 1)$, (3606303333486213243396180:808584977369673217366137: 1), (3183425943033245702793000: 1634707051192673592790909: 1)], [(6363393546423453606183150: $140303416078458529993641: 1)$, (3336123093276273243426064: $2573772106825497898723330: 1$ ), (3063305792883095612702910: $863533335479157004799454: 1)]$, [(6453423606303273456153030: 1145690416667479881785989:1), (3246123003216153123305730: $3337073052674808546802738: 1)$, (2732975702793005732823035: $150047494182361002398824: 1)]$, [(6273243426153123306273152: $3115488627365979385578692: 1)$, (3366183063275912883065400: $2931432536768990021556017: 1)$, (2492705642732945913003210: $1951224737429512321435284: 1)]$, [(6453423606153123305882850: $3671227255711398080608933: 1)$, (3035852823005912883066060 : $20719707193914097368399: 1)$, (3183306303423545852973090: 2363483130193579751119724:1)], [(6333363516093063245912880: 1619542044935651323570932: 1), (3065912883065942973126000: 178293515027268779112948: 1), (3123246213333455912943090: $4193464944833841470814307: 1)]$ [(6183213365972973215882881: $494038131959205661519572: 1)$, (3125972943186063033276032: $4537586987392051618798923: 1)$, (3063216033063215942973121: $3624192400884451079634437: 1)]$

Figure 12. The decrypted sequence of points

So, when an image is so encrypted, the original image can be reconstituted. The encrypted points can be decrypted as presented in II.A.1 and II.A.2. Then, by applying the inverse operations described in III.B and III.C, the matrix containing the initial pixel values is recovered. Finally, the reconstituted image is obtained by repeating the same process for all the blocks of the image.

\title{
B. Discussions
}

Table 1 below presents a comparison between the crypto-compression schemes implemented here and some known schemes (JPEG-AES [15], FMT-AES [4] and JPEG-EC-LFSR [6]). To compute the execution time, we used the SageMath function cputime() [17]. For the compression rate, the below formula has been used [4]: 
Vol. 8, Issue 5, May 2019

$$
t=1-\frac{\text { Size }_{\text {final }}}{\text { Siza } a_{\text {initial }}}
$$

For image quality, we considered the percentage of pixels not modified during the process [4]. In Table 1, "with losses" refers to an image quality less than $100 \%$ after reconstitution, while "lossless" indicates a $100 \%$ image quality.

Table 1. Comparison with some encryption- description systems

\begin{tabular}{|l|c|c|c|}
\hline "lena" & Quality & Time(s) & Rate \\
\hline Original image & $/$ & $/$ & $/$ \\
\hline JPEG-AES & with losses & 11.15 & $84 \%$ \\
\hline FMT-AES & with losses & 3.37 & $86 \%$ \\
\hline JPEG-EC-LFSR & with losses & 13.47 & $84 \%$ \\
\hline EC-MO & lossless & 137.95 & $87.5 \%$ \\
\hline EC-ElGamal & lossless & 1.09 & $87.5 \%$ \\
\hline
\end{tabular}

From table 1, it appears clearly that ElGamal based crypto-compression is better than the others. Indeed, it offers the smallest execution time for a better compression rate and an optimal quality. The great execution time taken by the Massey-Omura encryption is explained by the fact that in each step of the encryption, a multiplication of a point of $E$ by an integer is performed instead of a simple addition for ElGamal.

\section{CONCLUSION AND PERSPECTIVES}

Two crypto-compression schemes based on elliptic curves have been presented. Compared to others cryptocompression schemes, the results obtained here offer a better quality since the process is lossless, and a greater compression gain. The execution time is also clearly better with the ElGamal based encryption-compression. So, processing images as points of an elliptic curve improves their crypto-compression. Others operations on images (segmentation, watermarking, etc.) may also be improved by seeing images as points on elliptic curves.

\section{REFERENCES}

[1]. Bauer B., Donat-Bouillud P. and Durand V., Courbes elliptiques et cryptographie, Cours ENS Rennes, 2011.

[2]. Benabdellah M, Majid H.M., Zahid N., Regragui F. and Bouyakhf E.H., Encryption-Compression of still images using the FMT transformation and the DES algorithm, International Journal of Computer Sciences and Telecommunications, No. 4, 2006.

[3]. Benabdellah M, Majid H.M., Zahid N., Re- gragui F. and Bouyakhf E.H., Encryption- compression of images based on FMT and AES algorithm, International Journal Applied Mathematical Sciences, Vol. 1, 2007.

[4]. Bebabdellah M., Outils de compression et de crypto-compression: applications aux images fixes et vidéo, Thèse de doctorat, Université Mohammed V-Agdal, 2007.

[5]. Bos J. W., Halderman J. A., Heninger N.,Moore J., Naehrig M.and Wustrow E., Elliptic Curve Cryptography in Practice, IACR, 2013.

[6]. Cidjeu D.D. and Tieudjo D., Image crypto-compression based on Elliptic Curves and Linear Feedback Shift Register (LFSR), International Journal of Computer Science and Telecommunications, Vol. 9, Issue 7, pp 7-13, 2018.

[7]. Ftérich S. and Ben Amar C., Crypto- compression d'images fixes par la méthode de Quadtree optimisée et AES, CORESA, 2004.

[8]. Jalel H., Mohamed A., Ben F., Mounir S. and Abdennaceur K., Crypto-compression of images based on chaos, 6th International Con-ference on Sciences of Electronics, Technologies of Information and Telecommunications (SETIT), Sousse, pp. 344-350, 2012.

[9]. Koblitz N., A Course in Number Theory and Cryptography - 2nd ed., Springer-Verlag, 1994.

[10]. Martinez V.G., Encinas L.H. and Avila C.S., A Survey of the Elliptic Curve Int grated Encryption Scheme, Journal of Com- puter Science and Engeneering, Vol 2, 2010.

[11]. Masmoudi A., Puech W., Lossless chaos- based crypto-compression scheme for image protection, ITE Image Processing, vol 8, 2014.

[12]. Meraoubi H., Brahimi Z., Ait Saadi K. et Zemouri A. , Un système de crypto- compression des images médicales basé sur la DCT $2 \times 2-I D S$ et l'AES, 1st ISESC, 2005.

[13]. Puech W. and Rodrigues J.M., A new crypto-watermarking method for medical images safe transfer, 12th European Signal Pro- cessing Conference, Vienna, pp. 1481-1484, 2004.

[14]. Puech W. et Rodrigues J.M., Crypto-Compression of medical images by selective encryption of DCT, In EUSIPCO’05, Antalya, 2005.

[15]. Puech W., Rodrigues J.M. et Develay- Morice J.E., Transfert sécurisé d'images médicales par codage conjoint : cryptage sélectif par AES en mode par flot et compression JPEG, Traitement du signal (TS), numéro spécial Traitement du signal appliqué à la cancérologie, vol. 23, 5, 2006.

[16]. Puech W. et Coatrieux G., Codage hy-bride - cryptage-marquage-compression pour la sécurisation de l'information médicale, In Compression des images et des signaux médicaux (chap. 10), Lavoisier, IC2, pp.269-298, 2007.

[17]. Stein W., Sage for Power Users, Computer Aided Mathematics, 2012.

[18]. Xingyuan W., Xiaojuan W., Jianfeng Z. and Zhenfeng Z., Chaotic encryption algorithm based on alternant of stream cipher and block cipher, Nonlinear Dynamics, vol 63, Issue 4, pp 587-597, 2011. 